\title{
Random Finite Element Computation Analysis of Deformation of Multi-pivot Foundation Pit
}

\author{
Yang Ming ${ }^{1}$,Wang Jun $^{1}$,Wang Zhengxiang ${ }^{1}$,Wu Chaofeng ${ }^{2}$ \\ ${ }^{1}$ The 1st Geological Brigade of Jiangsu Geology and Mineral Exploration Bureau ， Nanjing \\ 210041 , China \\ ${ }^{2}$ Zhejiang Electric Power Design Institute Hangzhou 310014, China
}

Keywords: Multi-support, Foundation pit, Finite element.

\begin{abstract}
The paper selects shear strength index of soil as the random variable, and applies elastic foundation beam system finite element method and load incremental method to establish random finite element computing model of multi-pivot piling wall supporting foundation pit deformation and deduct the partial derivative of foundation pit deformation for random variable. Combined with the improved the first-order second-moment method, the paper analyzes the reliability of multi-pivot piling wall supporting foundation pit deformation for providing theoretical and research basis for the design method of deep excavation engineering based on reliability theory.
\end{abstract}

\section{Introduction}

With rapid development of the construction of high-rise buildings and municipal engineering in cities in China, there are more and more deep excavation engineering, and the cutting depth is greater and greater. In order to ensure the stability of foundation pit in construction and reduce the influence of foundation pit excavation on the surrounding buildings, roads and underground engineering facilities, the supporting structure system with inner support is generally used in urban deep foundation pit excavation such as campshed+pre-stressed anchor supporting system, campshed+inner supporting system and underground continuous wall+inner supporting system. We call the supporting system multi-pivot piling wall supporting system.

For the multi-pivot piling wall supporting system, the constant value design method based on security coefficient is generally used. Some scholars have made initial exploration for the reliability of single-pivot piling wall supporting system, but there is no research on the analysis of reliability of multi-pivot piling wall supporting system at home and abroad. The computation method has the disadvantages of large computation amount and low computation efficiency, so the practicality in engineering is worse.

\section{Finite Element Method}

For analyzing deformation of foundation pit and internal force of supporting structure, finite element method has the incomparable advantages. The method can simulate the actual excavation process of foundation pit, and fully considers the influence of the deformation of inner support and supporting structure on internal force of structure and deformation, so it is widely applied in the design computation of foundation pit engineering.

According to the characteristics of foundation pit engineering, finite element method can be divided into space finite element method, plane finite element method and bar-system finite element method. Space finite element method and plane finite element method uses continuum mechanics as the theoretical basis, and divide soil and supporting structure into computation units. In computation, the soil and supporting structure can flexibly apply different constitutive models, so the method is perfect theoretically. However, in application process, it has the disadvantages of complicated finite element procedure, large computation amount and low efficiency, so the method is not generally applied in engineering practice. 
Bar-system finite element method can use foundation pit supporting structure as elastic foundation beam based on elastic resistance method. And the function of the surrounding soil for supporting structure is replaced by soil pressure and soil spring, which avoids the disadvantage of the above finite element method. The model is simple, the computation is easy and the calculation results are ideal. So the method not only is simple and practical, but also can solve the actual engineering problems.

Based on the above analysis, the paper combines bar-system finite element method and elastic foundation beam method, simulates the actual excavation process of multi-pivot piling wall supporting foundation fit, fully considers the common function of soil, supporting structure and support, and establishes the computation model of inner force of supporting structure and deformation under different working conditions in the excavation process of foundation pit.

\section{Random Finite Element Computation Model of Deformation of Multi-pivot Foundation Pit}

For multi-pivot filing wall supporting foundation pit, soil excavation is the first, and the erection of inner support (or anchor stock) is the second. That is, before the erection of support (or anchor stock), the supporting structure has initially deformed, and the initial deformation can't be neglected in the design of foundation pit. In finite element analysis of multi-pivot piling wall supporting foundation pit, load full dose method and incremental method has the same calculation results. However, the analysis of load full-dose method needs to consider the inheritance of working conditions, and the initial displacement of the supporting side needs to be corrected, which makes the computation complicated and makes computer calculation program difficult. And applying load incremental method for analysis is easy and simple.

For the selection of random variables, the paper only selects the internal friction angle $\varphi_{i}$ and cohesion $c_{i}$ of soil as random variables, and the other variables are constants. There are $m$ soil layers in the range of foundation pit excavation, there are $2 m$ random variables, $\left(c_{1}, \varphi_{1}, c_{2}, \varphi_{2}, \cdots, c_{m}, \varphi_{m}\right)$. And all random variables meet normal distribution. In order to make expression easy, the random variables are expressed by $\left(X_{1}, X_{2}, X_{3}, \cdots, X_{2 m}\right)$.

In the computation, the unit wide wall is selected as mechanical analysis object, and it is divided into $n$ - 1 computation elements. The length of each computation elements is the same, and is expressed by $l_{e}$. The first inner support is located in the second node, the second inner support is placed in the sixth node, and the depth of foundation pit excavation is $H$.

The first working condition (the first excavation). For the first excavation, the working face of the first inner support is excavated. The first inner support is located in the second 2, so the depth of the first excavation is $H_{1}=2 l_{e}$, which means to excavate out the third node. And two soil springs are dig out, and the computation sketch is shown in Fig. 1. 


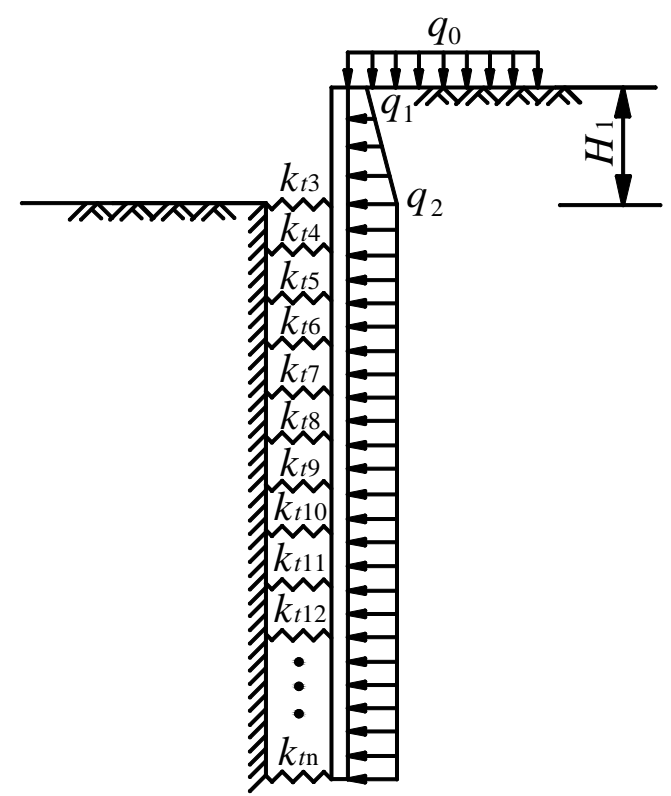

Fig. 1 Calculation diagram of the first working condition

Computation model of incremental method. The stiffness matrix $\boldsymbol{K}_{\mathbf{1}}$ of the supporting structure under the first working condition achieved by the correction of initial stiffness matrix $\boldsymbol{K}_{\mathbf{0}}$ is

$$
\begin{aligned}
& \boldsymbol{K}_{\mathbf{1}}=\left[\begin{array}{cccccccccc}
k_{11} & k_{12} & 0 & 0 & 0 & 0 & 0 & \cdots & 0 & 0 \\
k_{21} & k_{22} & k_{23} & 0 & 0 & 0 & 0 & \cdots & 0 & 0 \\
0 & k_{32} & k_{33}^{\prime} & k_{34} & 0 & 0 & 0 & \cdots & 0 & 0 \\
0 & 0 & k_{43} & k_{44}^{\prime} & k_{45} & 0 & 0 & \cdots & 0 & 0 \\
0 & 0 & 0 & k_{54} & k_{55}^{\prime} & k_{56} & 0 & \cdots & 0 & 0 \\
0 & 0 & 0 & 0 & k_{65} & k_{66}^{\prime} & k_{67} & \cdots & 0 & 0 \\
0 & 0 & 0 & 0 & 0 & k_{76} & k_{77}^{\prime} & \cdots & 0 & 0 \\
\vdots & \vdots & \vdots & \vdots & \vdots & \vdots & \vdots & \ddots & \vdots & \vdots \\
0 & 0 & 0 & 0 & 0 & 0 & 0 & \cdots & k_{(n-1),(n-1)}^{\prime} & k_{(n-1), n} \\
0 & 0 & 0 & 0 & 0 & 0 & 0 & \cdots & k_{n,(n-1)} & k_{n n}^{\prime}
\end{array}\right] \\
& \text { In the formula, } k_{i i}^{\prime}=\left[\begin{array}{cc}
\frac{24 E I}{l_{e}^{3}}+k_{t i} & 0 \\
0 & \frac{8 E I}{l_{e}}
\end{array}\right] \quad(i=3,4, \cdots, n-1) \text {; } \\
& k_{n n}^{\prime}=\left[\begin{array}{cc}
\frac{12 E I}{l_{e}^{3}}+k_{t n} & -\frac{6 E I}{l_{e}^{2}} \\
-\frac{6 E I}{l_{e}^{2}} & \frac{4 E I}{l_{e}}
\end{array}\right] ;
\end{aligned}
$$

And $k_{t i}(i=3,4,5, \cdots, n)$ means the elastic stiffness coefficient of the i soil spring.

The integral stiffness equation of the supporting structure under the first working condition is

$$
K_{1} \Delta_{1}=P_{1}
$$

The column vector $\boldsymbol{\Delta}_{\mathbf{1}}$ of the displacement increment is

$$
\boldsymbol{\Delta}_{\mathbf{1}}=\left[\begin{array}{lllllllll}
\Delta y_{1}^{1} & \Delta \theta_{1}^{1} & \Delta y_{2}^{1} & \Delta \theta_{2}^{1} & \Delta y_{3}^{1} & \Delta \theta_{3}^{1} & \cdots & \Delta y_{n}^{1} & \Delta \theta_{n}^{1}
\end{array}\right]^{T}
$$

The column vector $\boldsymbol{P}_{\mathbf{1}}$ of the equivalent load of the node is 


$$
\boldsymbol{P}_{\mathbf{1}}=\left[\begin{array}{lllllllll}
f_{1}^{1} & m_{1}^{1} & f_{2}^{1} & m_{2}^{1} & f_{3}^{1} & m_{3}^{1} & \cdots & f_{n}^{1} & m_{n}^{1}
\end{array}\right]^{T}
$$

Partial derivative of displacement increment for random variables

From formula (2), we can get

$$
\Delta_{1}=K_{1}^{-1} P_{1}
$$

In the formula, $K_{1}^{-1}$ is the inverse matrix of the integral stiffness matrix $K_{1}$.

Both sides of formula (5) solves partial derivative of random variable ${ }^{X_{i}}$ at the same time, which can get

And the expansion is

$$
\frac{\partial \Delta_{1}}{\partial X_{i}}=K_{1}^{-1} \frac{\partial P_{1}}{\partial X_{i}}
$$

$$
\left[\begin{array}{c}
\frac{\partial \Delta y_{1}^{1}}{\partial X_{i}} \\
\frac{\partial \Delta \theta_{1}^{1}}{\partial X_{i}} \\
\frac{\partial \Delta y_{2}^{1}}{\partial X_{i}} \\
\frac{\partial \Delta \theta_{2}^{1}}{\partial X_{i}} \\
\vdots \\
\frac{\partial \Delta y_{n}^{1}}{\partial X_{i}} \\
\frac{\partial \Delta \theta_{n}^{1}}{\partial X_{i}}
\end{array}\right]=\left[\begin{array}{cccccccccc}
k_{11} \\
k_{21} \\
k_{12} & k_{22} & k_{23} & 0 & 0 & 0 & 0 & \cdots & 0 & 0 \\
0 & k_{32} & k_{33}^{\prime} & k_{34} & 0 & 0 & 0 & \cdots & 0 & 0 \\
0 & 0 & k_{43} & k_{44}^{\prime} & k_{45} & 0 & 0 & \cdots & 0 & 0 \\
0 & 0 & 0 & k_{54} & k_{55}^{\prime} & k_{56} & 0 & \cdots & 0 & 0 \\
0 & 0 & 0 & 0 & k_{65} & k_{66}^{\prime} & k_{67} & \cdots & 0 & 0 \\
0 & 0 & 0 & 0 & 0 & k_{76} & k_{77}^{\prime} & \cdots & 0 & 0 \\
\vdots & \vdots & \vdots & \vdots & \vdots & \vdots & \vdots & \ddots & \vdots & \vdots \\
0 & 0 & 0 & 0 & 0 & 0 & 0 & \cdots & k_{(n-1),(n-1)}^{\prime} & k_{(n-1), n} \\
0 & 0 & 0 & 0 & 0 & 0 & 0 & \cdots & k_{n,(n-1)} & k_{n n}^{\prime}
\end{array}\right]^{-1}\left[\begin{array}{c}
\frac{\partial f_{1}^{1}}{\partial X_{i}} \\
\frac{\partial m_{1}^{1}}{\partial X_{i}} \\
\frac{\partial f_{2}^{1}}{\partial X_{i}} \\
\frac{\partial m_{2}^{1}}{\partial X_{i}} \\
\vdots \\
\frac{\partial f_{n}^{1}}{\partial X_{i}} \\
\frac{\partial m_{n}^{1}}{\partial X_{i}}
\end{array}\right]
$$

Partial derivative of counter force of soil spring for random variables

Under the first working condition, the counter force increment $\Delta N_{k}^{1}$ of soil spring is

$$
\Delta N_{k}^{1}=k_{t k} \Delta y_{k}^{1} \quad(k=3,4,5, \cdots, n)
$$

In the formula, $\Delta N_{k}^{1}-$ the counter force of the $\mathrm{k}$ soil spring under the first working condition, $\mathrm{kN}$;

$\Delta y_{k}^{1}$-the deformation increment of the k soil spring under the first working condition, $\mathrm{m}$;

$$
k_{t k} \text {-the stiffness coefficient of the } \mathrm{k} \text { soil spring, } \mathrm{kN} / \mathrm{m} \text {. }
$$

And the total counter force $N_{k}^{1}$ of soil spring is

$$
N_{k}^{1}=\Delta N_{k}^{1}=k_{t k} \Delta y_{k}^{1} \quad(k=3,4,5, \cdots, n)
$$

The partial derivative of the total counter force $N_{k}^{1}$ of soil spring for the random variable $X_{i}$ is

$$
\frac{\partial N_{k}^{1}}{\partial X_{i}}=k_{t k} \frac{\partial \Delta y_{k}^{1}}{\partial X_{i}} \quad(k=3,4,5, \cdots, n)
$$

Partial derivative of equivalent load of nodes for random variables

(1) The partial derivative of the equivalent load of the node that the first soil pressure is greater than zero unit for the random variable $X_{i}$ is 


$$
\left\{\begin{array}{l}
\frac{\partial f_{k}^{1}}{\partial X_{i}}=\frac{l_{e}}{20}\left(3 \frac{\partial q_{k+1}}{\partial X_{i}}+7 \frac{\partial q_{k}}{\partial X_{i}}\right) \\
\frac{\partial m_{k}^{1}}{\partial X_{i}}=\frac{l_{e}^{2}}{60}\left(2 \frac{\partial q_{k+1}}{\partial X_{i}}+3 \frac{\partial q_{k}}{\partial X_{i}}\right)
\end{array}\right.
$$

(2)The partial derivative of the equivalent load of any middle node for the random variable $X_{i}$ is

$$
\left\{\begin{array}{l}
\frac{\partial f_{k}^{1}}{\partial X_{i}}=\frac{l_{e}}{20}\left(3 \frac{\partial q_{k-1}}{\partial X_{i}}+7 \frac{\partial q_{k}}{\partial X_{i}}+7 \frac{\partial q_{k}^{\prime}}{\partial X_{i}}+3 \frac{\partial q_{k+1}}{\partial X_{i}}\right) \\
\frac{\partial m_{k}^{1}}{\partial X_{i}}=\frac{l_{e}^{2}}{60}\left(2 \frac{\partial q_{k-1}}{\partial X_{i}}+3 \frac{\partial q_{k}}{\partial X_{i}}+3 \frac{\partial q_{k}^{\prime}}{\partial X_{i}}+2 \frac{\partial q_{k+1}}{\partial X_{i}}\right)
\end{array}\right.
$$

(3)The partial derivative of the equivalent load of the n node for random variable ${ }^{X_{i}}$ is

$$
\left\{\begin{array}{l}
\frac{\partial f_{n}^{1}}{\partial X_{i}}=\frac{l_{e}}{20}\left(3 \frac{\partial q_{n-1}}{\partial X_{i}}+7 \frac{\partial q_{n}}{\partial X_{i}}\right) \\
\frac{\partial m_{n}^{1}}{\partial X_{i}}=-\frac{l_{e}^{2}}{60}\left(2 \frac{\partial q_{n-1}}{\partial X_{i}}+3 \frac{\partial q_{n}}{\partial X_{i}}\right)
\end{array}\right.
$$

The partial derivative of the active soil pressure strength $\mathrm{q}$ of each node for the random variable $^{X_{i}}$ is

$$
\begin{cases}\frac{\partial q}{\partial c_{i}}=-2 \tan \left(45^{\circ}-\frac{\varphi_{j}}{2}\right) & (i=j) \\ \frac{\partial q}{\partial c_{i}}=0 & (i \neq j) \\ \frac{\partial q}{\partial \varphi_{i}}=\sec ^{2}\left(45^{\circ}-\frac{\varphi_{j}}{2}\right)\left[2 \sum \gamma h \tan \left(45^{\circ}-\frac{\varphi_{j}}{2}\right)-2 c_{j}\right] & (i=j) \\ \frac{\partial q}{\partial \varphi_{i}}=0 & (i \neq j)\end{cases}
$$

The second working condition (erecting the first support and pre-add strutting axial force $F_{\mathrm{N} 1}$ ). Based on the first working condition, the first inner support (the elastic stiffness coefficient is $k_{z 1}$ ) is erected, and the axial force $F_{\mathrm{N} 1}$ (a constant) is pre-added. The computation sketch of the load increment of the working condition is shown in Figure 2. 


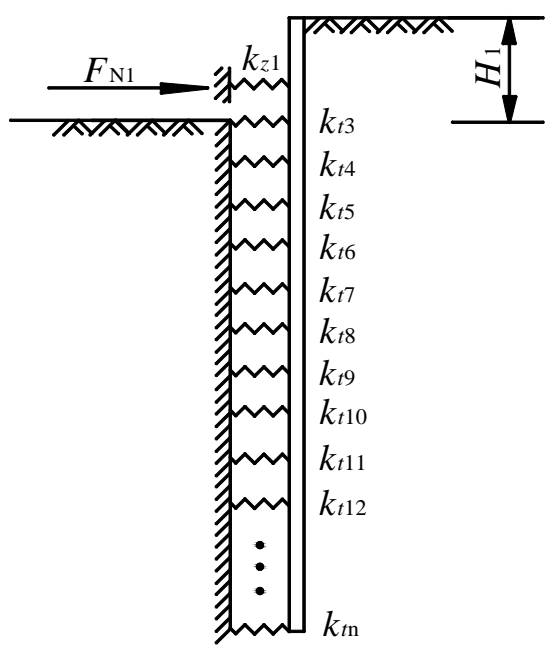

Fig. 2 Calculation diagram of the second working condition Computation model of increment method

The integral stiffness matrix $\boldsymbol{K}_{2}$ of the supporting structure under the second working condition which is achieved by the correcting of the integral stiffness matrix ${ }^{\mathbf{1}}$ under the first working condition is

In the formula, $k_{22}^{z 1}=\left[\begin{array}{cc}\frac{24 E I}{l_{e}^{3}}+k_{z 1} & 0 \\ 0 & \frac{8 E I}{l_{e}}\end{array}\right]$;

$$
\boldsymbol{K}_{2}=\left[\begin{array}{cccccccccc}
k_{11} & k_{12} & 0 & 0 & 0 & 0 & 0 & \cdots & 0 & 0 \\
k_{21} & k_{22}^{z 1} & k_{23} & 0 & 0 & 0 & 0 & \cdots & 0 & 0 \\
0 & k_{32} & k_{33}^{\prime} & k_{34} & 0 & 0 & 0 & \cdots & 0 & 0 \\
0 & 0 & k_{43} & k_{44}^{\prime} & k_{45} & 0 & 0 & \cdots & 0 & 0 \\
0 & 0 & 0 & k_{54} & k_{55}^{\prime} & k_{56} & 0 & \cdots & 0 & 0 \\
0 & 0 & 0 & 0 & k_{65} & k_{66}^{\prime} & k_{67} & \cdots & 0 & 0 \\
0 & 0 & 0 & 0 & 0 & k_{76} & k_{77}^{\prime} & \cdots & 0 & 0 \\
\vdots & \vdots & \vdots & \vdots & \vdots & \vdots & \vdots & \ddots & \vdots & \vdots \\
0 & 0 & 0 & 0 & 0 & 0 & 0 & \cdots & k_{(n-1),(n-1)}^{\prime} & k_{(n-1), n} \\
0 & 0 & 0 & 0 & 0 & 0 & 0 & \cdots & k_{n,(n-1)} & k_{n n}^{\prime}
\end{array}\right]
$$

In the integral stiffness equation of the supporting structure under the second working condition is

$$
K_{2} \Delta_{2}=P_{2}
$$

The column vector $\boldsymbol{\Delta}_{2}$ of the displacement increment is

$$
\boldsymbol{\Delta}_{2}=\left[\begin{array}{lllllllll}
\Delta y_{1}^{2} & \Delta \theta_{1}^{2} & \Delta y_{2}^{2} & \Delta \theta_{2}^{2} & \Delta y_{3}^{2} & \Delta \theta_{3}^{2} & \cdots & \Delta y_{n}^{2} & \Delta \theta_{n}^{2}
\end{array}\right]^{T}
$$

The column vector $\boldsymbol{P}_{2}$ of the equivalent load increment of the node is

$$
\boldsymbol{P}_{2}=\left[\begin{array}{lllllllll}
f_{1}^{2} & m_{1}^{2} & f_{2}^{2} & m_{2}^{2} & f_{3}^{2} & m_{3}^{2} & \cdots & f_{n}^{2} & m_{n}^{2}
\end{array}\right]^{T}
$$

In the formula, $f_{2}^{2}=-F_{N 1}$, and the others are zero.

Mean and variance of displacement increment

From formula (16), we can get

$$
\Delta_{2}=K_{2}^{-1} P_{2}
$$


In the formula, $\boldsymbol{K}_{2}^{-1}$ - the inverse matrix of the integral stiffness matrix $\boldsymbol{K}_{2}$.

Both sides of formula (19) solve derivative of random variable $X_{i}$ at the same time, which can get

$$
\partial P_{2} / \partial X_{i}=0, \text { so } \quad \begin{aligned}
& \frac{\partial \Delta_{2}}{\partial X_{i}}=K_{2}^{-1} \frac{\partial P_{2}}{\partial X_{i}} \\
& \frac{\partial \Delta y_{k}^{2}}{\partial X_{i}}=\frac{\partial \Delta \theta_{k}^{2}}{\partial X_{i}}=0
\end{aligned}
$$

From formula (19), we can get the horizontal displacement value $\Delta y_{k}^{2}$ (constant) of each node, and we can get the mean $\mu_{\Delta y_{k}^{2}}$ and the variance $\sigma_{\Delta y_{k}^{2}}^{2}$ of the horizontal displacement value $\Delta y_{k}^{2}$ of each node.

$$
\left\{\begin{array}{l}
\mu_{\Delta y_{k}^{2}}=\Delta y_{k}^{2} \\
\sigma_{\Delta y_{k}^{2}}^{2}=0
\end{array}\right.
$$

Partial derivative of counter force of soil spring for random variables

Under the second working condition, the increment $\Delta N_{k}^{2}$ of the counter force of soil spring is

$$
\Delta N_{k}^{2}=k_{t k} \cdot \Delta y_{k}^{2} \quad(k=3,4,5, \cdots, n)
$$

In the formula: $\Delta N_{k}^{2}$ - the increment of the counter force of the $\mathrm{k}$ soil spring under the second working condition, $\mathrm{kN}$;

$\Delta y_{k}^{2}$ - the displacement increment of the k node under the second working condition, $\mathrm{m}$;

$k_{t k}$-the stiffness coefficient of the $\mathrm{k}$ soil spring, $\mathrm{kN} / \mathrm{m}$.

And the total counter force of soil spring is

$$
N_{k}^{2}=N_{k}^{1}+\Delta N_{k}^{2}=k_{t k}\left(\Delta y_{k}^{1}+\Delta y_{k}^{2}\right) \quad(k=3,4,5, \cdots, n)
$$

The partial derivative of the total counter force $N_{k}^{2}$ of soil spring for random variable ${ }^{X_{i}}$ is

$$
\frac{\partial N_{k}^{2}}{\partial X_{i}}=k_{t k} \frac{\partial \Delta y_{k}^{1}}{\partial X_{i}}=\frac{\partial N_{k}^{1}}{\partial X_{i}} \quad(k=3,4,5, \cdots, n)
$$

\section{Computation of Reliability of Deformation of Foundation Pit}

The final horizontal deformation displacement of the $\mathrm{k}$ node of the supporting wall is

$$
y_{k}=\Delta y_{k}^{1}+\Delta y_{k}^{2}+\Delta y_{k}^{3}+\Delta y_{k}^{4}+\Delta y_{k}^{5}
$$

The failure function of the horizontal deformation displacement of the k node of the supporting wall is

$$
g_{k}=[y]-y_{k}=[y]-\left(\Delta y_{k}^{1}+\Delta y_{k}^{2}+\Delta y_{k}^{3}+\Delta y_{k}^{4}+\Delta y_{k}^{5}\right)
$$

In the formula, $[y]$ _the limiting value of the horizontal deformation of the supporting structure of the foundation pit, and can take $0.3 \% \sim 0.5 \%$ of the depth of foundation pit excavation.

The partial derivative of the failure function $g_{k}$ for the random variable $X_{i}$ is

$$
\frac{\partial g_{k}}{\partial X_{i}}=-\left(\frac{\partial \Delta y_{k}^{1}}{\partial X_{i}}+\frac{\partial \Delta y_{k}^{3}}{\partial X_{i}}+\frac{\Delta y_{k}^{5}}{\partial X_{i}}\right)
$$

\section{$g_{k}$ obeys normal distribution}

If $\Delta y_{k}^{1}, \Delta y_{k}^{2}, \Delta y_{k}^{3}, \Delta y_{k}^{4}$ and $\Delta y_{k}^{5}$ obeys normal distribution, from formula (27), we can know 
that the failure function $g_{k}$ obeys normal distribution. And the man of the failure function $g_{k}$ is

$$
\mu_{g_{k}}=[y]-\sum_{i=1}^{5} \mu_{\Delta y_{k}^{i}}
$$

The variance of $g_{k}$ is

$$
\sigma_{g_{k}}^{2}=\sum_{i=1}^{5} \sigma_{\Delta y_{k}^{i}}^{2}+\sum_{i=1}^{5} \sum_{j=1}^{5} \operatorname{cov}\left(\Delta y_{k}^{i}, \Delta y_{k}^{j}\right)
$$

In the formula, $\operatorname{cov}\left(\Delta y_{k}^{i}, \Delta y_{k}^{j}\right)$ - the covairance of random variable $\Delta y_{k}^{i}$ and $\Delta y_{k}^{j}$.

The reliability indicator $\beta_{k}$ of the horizontal deformation displacement of the $\mathrm{k}$ node of the supporting wall is

$$
\beta_{k}=\frac{\mu_{g_{k}}}{\sigma_{g_{k}}}=\frac{[y]-\sum_{i=1}^{5} \mu_{\Delta y_{k}^{i}}}{\sqrt{\sum_{i=1}^{5} \sigma_{\Delta y_{k}^{i}}^{2}+\sum_{i=1}^{5} \sum_{j=1}^{5} \operatorname{cov}\left(\Delta y_{k}^{i}, \Delta y_{k}^{j}\right)}}
$$

\section{$g_{k}$ is abnormal distribution}

When $g_{k}$ is abnormal distribution, the value of reliability indicator $\beta_{k}$ can't be achieved simply from the formula (31). And achieving the value of reliability indicator $\beta_{k}$ needs iterative solution.

$X^{*}=\left(X_{1}^{*}, X_{2}^{*}, X_{3}^{*}, \cdots, X_{2 m}^{*}\right)=\left(c_{1}^{*}, \varphi_{1}^{*}, c_{2}^{*}, \varphi_{2}^{*}, \cdots, c_{m}^{*}, \varphi_{m}^{*}\right)$ is design checking point. The failure function $g_{k}$ receives Taylor linear expansion at the design checking point $X^{*}$,

$$
Z_{k} \approx g_{k}\left(X_{1}^{*}, X_{2}^{*}, \ldots, X_{2 m}^{*}\right)+\left.\sum_{i=1}^{2 m}\left(X_{i}-X_{i}^{*}\right)\left(\frac{\partial g_{k}}{\partial X_{i}}\right)\right|_{X^{*}}
$$

The calculation process of the reliability indicator $\beta_{k}$ of the horizontal deformation displacement of the $\mathrm{k}$ node of the supporting wall is as follows.

(1) The initial value of $X^{*}$ and $\beta_{k}$ is $X^{*}=\mu_{X_{i}}$ and $\beta_{k}=3$.

(2) Based on Ranking active spoil pressure theory, the load increment $q_{k}^{i}$ of each node under working conditions is calculated, and formula (14) is used to figure out $\left.\frac{\partial q_{k}^{i}}{\partial X_{j}}\right|_{X=X^{*}}$

(3) Substituting $\left.\frac{\partial q_{k}^{i}}{\partial X_{j}}\right|_{X=X^{*}}$ into formula (11) $\sim(13)$ can get $\left.\frac{\partial f_{k}^{i}}{\partial X_{j}}\right|_{X=X^{*}}$ and $\left.\frac{\partial m_{k}^{i}}{\partial X_{j}}\right|_{X=X^{*}}$.

(4) Substituting $\left.\frac{\partial f_{k}^{i}}{\partial X_{j}}\right|_{X=X^{*}}$ and $\left.\frac{\partial m_{k}^{i}}{\partial X_{j}}\right|_{X=X^{*}}$ into formula (17) and (24) can get $\left.\frac{\partial \Delta y_{k}^{i}}{\partial X_{j}}\right|_{X=X^{*}}$.

(5) Substituting $\left.\frac{\partial \Delta y_{k}^{i}}{\partial X_{j}}\right|_{X=X^{*}}$ into formula (28) can get $\left.\frac{\partial g_{k}}{\partial X_{i}}\right|_{X=X^{*}}$.

(6) Substituting $\left.\frac{\partial g_{k}}{\partial X_{i}}\right|_{X=X^{*}}$ into formula (32) can figure out the sensitivity coefficient $\alpha_{i}$. 


$$
\alpha_{i}=\frac{\left.\sum_{i=1}^{2 m} \frac{\partial g_{k}}{\partial X_{i}}\right|_{X^{*}} \rho_{i j} \sigma_{X_{i}}}{\left[\left.\sum_{i=1}^{2 m} \sum_{j=1}^{2 m} \frac{\partial g_{k}}{\partial X_{i}} \cdot \frac{\partial g_{k}}{\partial X_{j}}\right|_{X^{*}} \rho_{i j} \sigma_{X_{i}} \sigma_{X_{j}}\right]^{\frac{1}{2}}}
$$

In the formula, $\rho_{i j}$ — the correlation coefficient of random variable $X_{i}$ and $X_{j}$.

(7) Substituting $\alpha_{i}$ and $\beta_{k}$ into formula (33) can figure out the new $X^{*}$.

$$
X_{i}^{*}=\mu_{X_{i}}-\beta_{k} \alpha_{i} \sigma_{X_{i}}
$$

(8) According to formula (34), the new $\beta_{k}$ is figured out.

$$
\beta_{k}=\frac{g_{k}\left(X_{1}^{*}, X_{2}^{*}, \cdots, X_{2 m}^{*}\right)+\left.\sum_{i=1}^{2 m}\left(\mu_{X_{i}}-X_{i}^{*}\right) \frac{\partial g_{k}}{\partial X_{i}}\right|_{X^{*}}}{\left.\sum_{i=1}^{2 m} \alpha_{i} \sigma_{X_{i}} \frac{\partial g_{k}}{\partial X_{i}}\right|_{X^{*}}}
$$

New $X_{i}^{*}$ and $\beta_{k}$ is the initial value, the step from (2) $\sim(8)$ is repeated until the difference of $\beta_{k}$ is less than the required calculation accuracy, which can get the final reliability indicator $\beta_{k}$ and the design checking point $X^{*}=\left(c_{1}^{*}, \varphi_{1}^{*}, c_{2}^{*}, \varphi_{2}^{*}, \cdots, c_{m}^{*}, \varphi_{m}^{*}\right)$.

The reliability indicator $\beta$ of the horizontal deformation displacement of supporting structure of foundation pit is

$$
\beta=\min \left\{\beta_{1}, \beta_{2}, \beta_{2}, \cdots, \beta_{n}\right\}
$$

The above calculation process is written into the calculation procedure, which can can realize iteration calculation by computers.

\section{Conclusions}

Based on finite element method and load increment method of elastic foundation beam system, the paper uses the computation model of elastic soil pressure,and establishes random finite element computation model of supporting structure deformation analysis under working conditions of multi-pivot piling wall supporting foundation pit. The shearing intensity of soil is selected as random variable to deduct the computation formula of partial derivative of horizontal displacement of supporting structure for random variables. And the paper uses the improves the first-order second-moment method to figure out the reliability indicator of horizontal deformation of foundation pit, which forms analysis method of horizontal deformation of multi-pivot piling wall supporting foundation.

Combined with the engineering projects, the paper analyzes the variable coefficient of shearing strength indicator of soil and the influence of position of soil layer on reliability indicator of horizontal deformation of foundation soil, and the research indicates that

(1) The reliability indicator $\beta$ of horizontal deformation of foundation pit reduces with the increase of the soil cohesion ${ }^{C}$ and the variance coefficient of internal friction angle $\varphi$. The variance coefficient of internal friction angle $\varphi_{\text {has greater influence on reliability indicator }} \beta$ of deformation of foundation pit, and the variance coefficient of cohesion ${ }^{C}$ has little influence on reliability indicator $\beta$.

(2) The place of soil layer in the excavation range of foundation pit has great influence on the reliability indicator $\beta$ of horizontal deformation of foundation pit, and the influence of the variance coefficient of shearing strength indicator on reliability indicator $\beta$ of horizontal deformation of 
foundation fit is greater than that of the soil layer under excavation face. For the soil above excavation face, the shearing strength indicator of thicker soil layer has greater influence on indicator indicator $\beta$ of horizontal deformation of foundation pit.

\section{References}

[1] Cao Cehui. Application of stochastic finite element method in the reliability analysis of bolt surrounding rock structure. The second national youth Symposium on rock mechanics and engineering. Beijing: 1999193-189.

[2] Tan Xiaohui. The study of slope stability reliability analysis methods. Journal of Chongqing University (NATURAL SCIENCE EDITION).2001,24 (6): 40-44.

King of the [3] state. The stability analysis of soil slope reliability index. Journal of HeFei University of Technology (NATURAL SCIENCE EDITION).2002,25 (3): 398 -402.

[4] Jia Houhua, he Huaijian. Fuzzy stochastic reliability analysis of slope stability. Rock and soil mechanics,.2003,24 (4): 657-660.

[5] Xu wenda. Analysis of slope of Monte Carlo finite element method based on reliable.2004. Journal of Fuzhou University, 32 (1): 68- 73

[6] Liu

Ming Wei, He Chunguang. The slope stability and reliability of the analysis based on Monte Carlo method. Journal of Chongqing Jianzhu University,.2001,23 (5): 96-99.

[7] Liu Xingjing. The vertical bearing capacity of single pile design and reliability estimation. Journal of Changsha Institute of traffic.2001,17 (1): 66-71.

[8] Liu Ning, Guo Zhichuan, Luo Boming. The calculation method of probability analysis of settlement and reliability. Chinese Journal of geotechnical engineering.2000,22 (2): 143-150.

[9]Casagrands A. Role of the calculated risk in earthwork and foundation engineering. Journal of the Soil Mechanics and Foundation Division.1965,91 (4):10-40P

[10]Lumb P. Statistical methods on soil investigation. Proc.5th Austrilia-New Zerland and Cont. On Application of Statistics and Probability in Soil and Structural Engineering. 1975(2):294-298P

[11]Harr M E. Reliability-based design in civil engineering. Mc Graw-hill,New York, 1977:56-57P

[12]Harr M E. Mechanics of particulate media-a probabilistic approach. Mc Graw -hill, New York,1997:34-36P

[13]Vanmarcke E H. Probabilistic modeling of soil profiles. J. Geotech. Eng. Div.,ASCE. 1977,103:1227-1246P

[14]Vanmarcke E H \& Fuleihsn N F. Probabilistic prediction of levee settlements. Proc. 2nd Int. Cont. On Application of Statistics and Probability in Soil and Structural Engineering, 1975(2):684-687P 